\title{
Linguistic Variables of Type-N. A Mathematical Model $l^{1}$
}

R.H.N. SANTIAGO2, Depto de Informática e Matemática Aplicada - DIMAp, UFRN, 59.072-970 Natal, RN, Brasil.

C. MAEDER, 3 , DFKI GmbH, Safe and Secure Cognitive Systems, EnriqueSchmidt Straße, 28359 Bremen, Germany.

\begin{abstract}
This work proposes a generalization for the notions of Linguistic Variables and Fuzzification Process. The idea is to provide a concept of fuzzy systems in which the linguistic terms of a linguistic variable are not necessarily connected with fuzzy sets of the same type. Those systems are called here Systems of Type-n. We introduce concepts like $n$-Homogeneous linguistic variables which emphasizes the ocurrence of different levels of uncertainty in such Fuzzy systems. The most important result is the statement of the General Fuzzification Expression (GFE) which establishes how the fuzzification process must be calculated in every system of type-n.
\end{abstract}

Keywords. Fuzzy Sets, Type-n Fuzzy sets, systems of type-n, n-homogeneous linguistic variables, heterogeneous linguistic variables.

\section{Introduction}

Since the work of Alan Turing [8] many mathematical models for machines have been proposed. Such models capture the complexity, power and goal of those artifacts; e.g. languages parsers, languages transducers [4], parallel machines (PRAM) [3], etc.

Fuzzy Expert Systems (FES) and Fuzzy Control Systems (FCS) are machines which have been largely used in Computing and Engineering and they have been traditionally [7] seen as machines which usually deal with uncertainty as values in the set $[0,1]$. Those machines will be called here as Systems of Type-0. However, the concept of Type-n fuzzy sets $[9,10,11]$ leads quickly to the possibility to generalize such systems to Systems of Type-n; namely, systems where the level of uncertainty are Fuzzy Sets of Type-n. However, it is worth to note that such generalization should maintain a certain homogeneity; namely every uncertainty value is a Fuzzy set of the same Type-n. So, another level of generalization would be machines which deals with different levels of uncertainty in the same linguistic

\footnotetext{
${ }^{1}$ This work was supported by CAPES and is part of the project BEX 1338/07-2.

${ }^{2}$ regivan@dimap.ufrn.br.

${ }^{3}$ Christian.Maeder@dfki.de.
} 
variable. The problem that arises is the following: How do we generalize max-min fuzzification $[1,2,5]$ to deal with different levels of uncertainty?

This is the aim of this paper, to provide a theoretical model for systems able to contain such aspects. A system with such characteristic will be called Heterogeneous Fuzzy Type-n System; on the other hand, systems with the same level of uncertainty - e.g. system where every value of uncertainty is a Type-1 fuzzy set will be called Homogeneous Fuzzy Type-n System. To apply fuzzification on different levels of uncertainty, there must exist operations of coercion between them. Aiming to give a rigorous semantics for such situation, we provide an alternative definition for Linguistic Variables which emphasizes that their linguistic terms can be linked to fuzzy sets with different levels of uncertainty - this will give rise to the notion of Heterogeneous/Homogeneous Linguistic Variables.

So, far from any comparison, this paper humbly makes the same work made by Turing at [8] in the sense that he provided a model for computers before their existence. That is, what we are trying to do here is to provide the first steps toward a model of General Fuzzy Machines, i.e. machines able to deal with different levels of uncertainty, even before their existence (but based on the possibility of that). This paper, however, neither model rules nor defuzzification in this context, this is a subject of another work; it is concerned with Linguistic Variables and how they are processed by fuzzification. To achieve that we provide an initial algebraic viewpoint for Fuzzy processing, where fuzzification is seen as an operation which transform linguistic variables. In this text the reader may find expressions like fuzzification process and fuzzification expression; the fuzzification expression is the expression (like Max-min) which defines the function fuzzification (the process).

This paper is structured in the following way: Section 2. proposes an alternative definition for linguistic variables. Section 3. describes the usual Fuzzy Systems which usually apply Max-min Fuzzification and establishes some arguments which will be generalized in section 4 .. Section 4 . is the heart of the paper, since it provides a mathematical way to gngeneralize usual Type-0 Fuzzy Systems. Section 5. generalizes Max-min Fuzzification to Type-n Systems by providing a coercion mechanism for its operands and giving rise to a generalized form of fuzzification called General Fuzzy Expression (GFE). The paper closes with some final remarks about the theme.

\section{2. $\quad$ Linguistic Variables}

Linguistic variables are the heart of fuzzy systems, since they provide the connection between fuzzy sets and linguistic terms. One standard definition for linguistic variables, found in [6], is

Definition 2.1. A linguistic variable is a quintuple $\langle x, T(x), U, G, M\rangle$; where: $x$ is the name of the variable; $T(x)$ is a set of terms which are values for the variable; $U$ is the universe of discourse which defines the characteristics of the variable; $G$ is a syntatic grammar which produces the terms in $T(x) ; M$ semantic rules which maps terms in $T(X)$ to fuzzy sets in $U$. 
In other words, linguistic variables are objects which allow the association between terms and elements of an universe together with a confidence about that.

In many applications, especially in Control Systems, linguistic variables are used to associate names to some notion of quantity. For example, a linguistic variable with terms like "Small", "Medium", etc are associated with real numbers. But, according to the previous definition, linguistic terms are not restricted refer quantities. In fact, linguistic variables can also be used in expert systems where terms can also denote objects with no relation to quantities.

Since linguistic variables state the association between terms and elements of the universe with a degree of confidence, the concept of confidence in an essential part in the definition of linguistic variables. However, it is worthy to note that the notion of confidence or uncertainty as values in the interval $[0,1]$ can be extended, and in the literature the corresponding concept for that is the notion of type-n fuzzy set [9]. The idea is: If the grade of membership $f(x)$ is a value in $[0,1]$, the corresponding fuzzy set " $f$ " is called of type-1, if the grade is a type-1 fuzzy set, then the corresponding fuzzy set is of type-2, and so on. Here we make the convention that an element $r \in[0,1]$ is also called a fuzzy set of type-0. Hence, following this way, the concept of type-n fuzzy sets allows that in the same linguistic variable different terms can be assigned to elements of the universe with different levels of uncertainty. This, together with the corresponding impact in fuzzification, as far as we know, are not treated in the literature. In what follows we provide a slight generalization for the notion of linguistic variables, in order to deal with this situation.

Definition 2.2 (Alternate Definition). A linguistic variable $\mathcal{L}$ is a structure

$$
\mathcal{L}=\left\langle N_{\mathcal{L}}, U_{\mathcal{L}}, G_{\mathcal{L}}, T_{\mathcal{L}}, M_{\mathcal{L}}\right\rangle,
$$

where

1. $N_{\mathcal{L}}$ is a string designating the name of the linguistic variable,

2. $U_{\mathcal{L}}$ is the universe of discourse which defines the characteristics of the variable;

3. $G_{\mathcal{L}}$ is a formal grammar [4] which generates the linguistic terms of $\mathcal{L}$;

4. $T_{\mathcal{L}}$ is a fuzzy set; which associates the elements generated by $G_{\mathcal{L}}$ to a type- $n$ fuzzy set; i.e. $T_{\mathcal{L}}$ is a function of the form $T_{\mathcal{L}}: L\left(G_{\mathcal{L}}\right) \rightarrow \mathbb{F} \mathbb{Z S E T}$, where $L\left(G_{\mathcal{L}}\right)$ is the formal language generated by $G_{\mathcal{L}}$ and $\mathbb{F} \mathbb{Z S E T}$ is the class of all type-0, type-1, etc fuzzy sets. The elements of $L\left(G_{\mathcal{L}}\right)$ are called linguistic values or linguistic terms, while the elements of the fuzzy set $T_{\mathcal{L}}$ are called fuzzy terms.

5. $M_{\mathcal{L}}$ is a, possibly empty, function $M_{\mathcal{L}}: L\left(G_{\mathcal{L}}\right) \rightarrow\left(U_{\mathcal{L}} \rightarrow \mathbb{F} \mathbb{Z} \mathbb{S E T}\right) ;$ i.e. a function which associates a linguistic term to a type-n fuzzy set with domain $U_{\mathcal{L}}$. The set $\left(U_{\mathcal{L}} \rightarrow \mathbb{F Z \mathbb { S E T }}\right)$ contains all the type-n fuzzy sets with domain $U_{\mathcal{L}}$.

When the context is clear we drop the subscripts $\mathcal{L}$. The class of all linguistic variables is denoted here by $\mathbb{L} \mathbb{V} \mathbb{R}$. 
In this paper we do not expose the structure of both $\mathbb{F} \mathbb{Z} \mathbb{E} \mathbb{T}$ and $\mathbb{L} \mathbb{V} \mathbb{R}$, this is subject of a future work which will deal with rules.

\section{Max-min Fuzzification in Systems of Type-0}

According to [7] p.51:

"To fuzzify has two meanings: (1) to transform a classical concept into a fuzzy concept; and (2) according to an input fuzzy set $A$, to find the confidence of fuzzy terms; i.e. the values $T_{\mathcal{L}}(x)$, for every $x \in L\left(G_{\mathcal{L}}\right)$."

We apply fuzzification in the second sense. Moreover, the same authors state:

"At the end of a sequence of rule firings in a fuzzy expert systems we may end up with a FUZZY CONCLUSION C THAT IS A LINGUISTIC VARIABLE, WHOSE VALUES HAVE BEEN ASSIGNED GRADES OF MEMBERSHIP."

In other words, the action of some parts of a fuzzy expert system can be seen as operations on linguistic variables. From this standpoint, we can model fuzzification as a process which assigns grades of membership to the linguistic terms; namely as a function which updates an input linguistic variable - this is the initial algebraic viewpoint mentioned in the introduction.

The fuzzifications which are commonly applied in many systems; are those which associate each $(t, u) \in L\left(G_{\mathcal{L}}\right) \times U_{\mathcal{L}}$ with values at $[0,1]$ — For example, the association: (HighFever, 39) $\mapsto 0.9$. Those systems are called here: Systems of Type-0; because the level of uncertainty belongs to $[0,1]$. This notion will be generalized in the next section to the notion of Systems of Type-n, in order to model systems which assign other levels of uncertainty to each $(t, u) \in L\left(G_{\mathcal{L}}\right) \times U_{\mathcal{L}}$.

Definition 3.1. Given a fuzzy system $\Sigma$ with a set $\Gamma_{\Sigma} \subseteq \mathbb{L} \mathbb{A} \mathbb{R}$ of linguistic variables, $\Sigma$ is a System of Type-0, if for every $\mathcal{L} \in \Gamma_{\Sigma}, t \in L\left(G_{\mathcal{L}}\right)$ and $u \in U_{\mathcal{L}}$, $M_{\mathcal{L}}(t)(u) \in[0,1]$.

Definition 3.2 (Type-0 Fuzzified Linguistic Variables). Let $\Sigma$ be a system of Type$0, \mathcal{L}=\left\langle N_{\mathcal{L}}, U_{\mathcal{L}}, G_{\mathcal{L}}, T_{\mathcal{L}}, M_{\mathcal{L}}\right\rangle$ a linguistic variable of $\Sigma$, and a value $A \in[0,1]$, a linguistic variable $\mathcal{L}^{A}=\left\langle N_{\mathcal{L}}, U_{\mathcal{L}}, G_{\mathcal{L}}, T_{\mathcal{L}^{\mathcal{A}}}, M_{\mathcal{L}}\right\rangle$ is a fuzzified linguistic variable from $A$ and $\mathcal{L}$, if for all $t \in L\left(G_{\mathcal{L}}\right)$ and $x \in U_{\mathcal{L}}$,

$$
T_{\mathcal{L}^{A}}(t)=\max \left(\min \left(M_{\mathcal{L}}(t)(x), A\right)\right) .
$$

The expression (3.1) is the usual max-min fuzzification largely used in fuzzy systems. It assigns grades of confidence to the linguistic terms of $\mathcal{L}$. This process could be roughly seen as an operation: $\varphi_{A}: \Sigma \rightarrow \Sigma$, where $\varphi(\mathcal{L})=\mathcal{L}^{A}$.

It is not difficult to view a value $A \in[0,1]$ as a function $C_{A}: B \rightarrow[0,1]$; where $B$ is a suitable set. In other words, it is always possible to lift a type-0 fuzzy set up to a type-1 fuzzy set. This can be done in the following way: 
Proposition 3.1. Given any set $B$ and an element $r \in[0,1]$, there is a fuzzy set $C_{r}: B \rightarrow[0,1]$, such that $C_{r}(b)=r ;$ for every $b \in B$.

Observe that the set $B$ can be a singleton set " $\{a\}$ " or a big product type " $\prod A_{j}$ ". This proposition leads to the next.

Proposition 3.2. In a system of type-0, since, during a fuzzification, an input $v \in[0,1]$ is a type-0 fuzzy set, then for every linguistic variable $\mathcal{L}$, both $A_{v}$ and $T_{\mathcal{L}^{A}}$ are type-1 fuzzy sets of the form $A_{v}: U_{\mathcal{L}} \rightarrow[0,1]$ and $T_{\mathcal{L}}: L\left(G_{\mathcal{L}}\right) \rightarrow[0,1]$.

Proof. Straightforward from equation (3.1) and proposition 3.1.

This result means that equation (3.1) in Type-0 systems can be faced as an operation on Type- 1 fuzzy sets. To see this, consider the infimum operation " $\wedge$ " as the pointwise extension of the minimum over $[0,1]$ to the class of type- 1 fuzzy sets $(U \rightarrow[0,1])$, then the equation 3.1 can be rewritten as

$$
T_{\mathcal{L}^{A}}(t)=\max \left\{\left(M_{\mathcal{L}}(t) \wedge A_{v}\right)(u) \mid u \in U\right\} .
$$

Hence the fuzzification on type-0 systems could be seen as an operation " $\varphi$ " such that $T_{\mathcal{L}^{A}}(t)=\varphi\left(A_{v}, M_{\mathcal{L}}(t)\right) ;$ where $A_{v}$ and $M_{\mathcal{L}}(t)$ are both of type $\left(U_{\mathcal{L}} \rightarrow[0,1]\right)$ and $T_{\mathcal{L}^{A}}$ is of type $\left(L\left(G_{\mathcal{L}}\right) \rightarrow[0,1]\right)$ and all of them are type- 1 fuzzy sets. Therefore, the usual max-min fuzzification can be seen as an operation on type-1 fuzzy sets.

The questions which naturally arise are the following:

1. The argument applied here can be generalized to systems in which the underlying uncertainties are of type-1, or type-2, etc? i.e. is it also possible to reduce such systems to systems which deals with type-1 fuzzy sets?

2. Is it possible to generalize the systems of type- 0 to systems in which different elements of the universe $u \in U_{\mathcal{L}}$ are associated with uncertainties of different levels (giving rise to the idea of heterogeneous systems)? Following this way, are they also possible to be reduced homogeneously to systems which deals with type-1 fuzzy sets? In this case how would the expression of Max-min fuzzification looks like?

The following section deals with such questions, and based on the known notion of fuzzy sets of type-n, it introduces both: the notion of Systems of Type-n - where the level of uncertainty is not restricted to type- 0 fuzzy sets - and the concept of heterogeneous fuzzy systems - where different elements of the universe $u \in U_{\mathcal{L}}$ are associated with uncertainties of different levels. This generalization is done in such a way that the present section is a special case of it.

\section{Systems of Type-n}

Definition 4.1 (Type-n Linguistic Variables and Systems of Type-n). A Type$n$ Linguistic Variable, $\mathcal{L}$, or a Linguistic Variable of Type-n, abbreviated 
by $\tau(\mathcal{L})=n$, is a linguistic variable such that there are $u \in U_{\mathcal{L}}$ and $t \in L\left(G_{\mathcal{L}}\right)$, where $M_{\mathcal{L}}(u)(t)$ is a type-n fuzzy set, and such that for any other type-k fuzzy set $M_{\mathcal{L}}\left(u^{\prime}\right)\left(t^{\prime}\right)$, where $u^{\prime} \in U_{\mathcal{L}}$ and $t^{\prime} \in L\left(G_{\mathcal{L}}\right), k \leq n$. A Fuzzy system $\Sigma$ is called a Fuzzy System of Type-n, if for all of its linguistic variables $\mathcal{L}_{1}, \ldots, \mathcal{L}_{k}$, $\max \left(\tau\left(\mathcal{L}_{1}\right), \ldots, \tau\left(\mathcal{L}_{k}\right)\right)=n$. A linguistic variable, $\mathcal{L}$, is $n$-homogeneous if $M_{\mathcal{L}}(u)(t)$ is a type-n fuzzy set, for every $(u, t) \in U_{\mathcal{L}} \times T_{\mathcal{L}}$; otherwise it is called heterogeneous. A n-homogeneous system is that in which every linguistic variable is n-homogeneous; otherwise it is called heterogeneous system.

According to this definition, usual systems of type-0 are homogeneous.

The next lemma answers the previous first question. Based on a set theory fact it shows that for every type-n fuzzy set there is an equivalent type- 1 fuzzy set, consequently, is it possible to reduce every system of type-n a system of type- 1 .

Lemma 4.1. Given a type-n fuzzy set $f: A_{1} \rightarrow\left(A_{2} \rightarrow\left(\ldots\left(A_{n} \rightarrow[0,1]\right) \ldots\right)\right)$, also written without parentheses as $f: A_{1} \rightarrow A_{2} \rightarrow \ldots A_{n} \rightarrow[0,1]$, there is an equivalent fuzzy set

$$
U C(f): A_{1} \times A_{2} \times \ldots A_{n} \rightarrow[0,1] .
$$

Proof. Since $\mathbb{S E T}$ is a cartesian closed category, $U C(f)\left(a_{1}, \ldots, a_{n}\right)$ is the uncurried version of $f$.

Corollary 4.1.1. Every type-n fuzzy set $(n>1)$ can be replaced by its uncurried counterpart " $U C(f)$ "; which is a type-1 fuzzy set.

Proposition 4.1. For every type-n fuzzy set, $n \in \mathbb{N}$, there is an equivalent type-1 fuzzy set.

Proof. It is enough to prove for $n=0$, for we have previously proved for $n>$ 1. According to proposition 3.1 , every real number $r \in[0,1]$ can be functionally represented by a function $C_{r}$.

Therefore, the results stated here show that every level of uncertainty can be reduced to a Type-1 fuzzy set. Hence, any system of any type can be reduced to a Type-1 system. Also note that, since the notion of uncertainty/confidence is not restricted to the set $[0,1]$, it is fairly possible to have a system with some linguistic variable such that for some $t \in L\left(G_{\mathcal{L}}\right)$ and $u \in U_{\mathcal{L}}, M_{\mathcal{L}}(t)(u)$ are not fuzzy sets of the same level, i.e. functions of the same type; for example, it is possible that for some $(s, v) \in L\left(G_{\mathcal{L}}\right) \times U_{\mathcal{L}}$ the associated notion of uncertainty, $M_{\mathcal{L}}(s)(v)$, be a type-0 fuzzy set, i.e. a number at $[0,1]$, and some other $(t, u) \in L\left(G_{\mathcal{L}}\right) \times U_{\mathcal{L}}$, $M_{\mathcal{L}}(t)(u)$ is a type- 1 fuzzy set. We have proved that those fuzzy sets can all be reduced to Type-1 fuzzy sets, but with different domains, therefore it is reasonable to ask:

1. "How do we calculate the fuzzification in such situation?"

or in other words, how do we generalize max-min fuzzification to deal with such situation? 


\section{Sup-inf Fuzzification in Systems of Type-n}

Analysing the Max-min expression at equation (3.2) we can observe some facts:

1. the expression " $\max \left\{\left(M_{\mathcal{L}}(t) \wedge A\right)(u) \mid u \in U\right\}$ " imposes that $A(u)$ and $M_{\mathcal{L}}(t)(u)$ should have the same type.

2. The type of $M_{\mathcal{L}}(t)(u)$ only depends on $u \in U_{\mathcal{L}}$, because for any two $t_{1}, t_{2} \in$ $L\left(G_{\mathcal{L}}\right)$ the types of $M_{\mathcal{L}}\left(t_{1}\right)(u)$ and $M_{\mathcal{L}}\left(t_{2}\right)(u)$ must be identical to $A_{v}(u)$.

3. Since, for each $u \in U_{\mathcal{L}}$ infimum operations can produce values of different types, the generalization of the external "max" function at equation (3.2) presupposes coercion operations on its operands before its generalization to a supremum operation (written as $\vee$ ).

Therefore, equation (3.2) must be generalized to an equation like

$$
T_{\mathcal{L}^{A}}(t)=\bigvee_{u \in U_{\mathcal{L}}}\left[\left(M_{\mathcal{L}}(t) \wedge A\right)(u)\right]
$$

At this point, this equation does not state explicit how coercions must be applied before the calculation of supremum. In the next section we provide such coercions and the resulting version for equation (5.1).

\subsection{Coersions and general fuzzification expression}

Previously, item 4 stated the requirements for coersions to evaluate Sup-inf fuzzification. In this subsection we provide some detailed explanation of that.

In order to simplify the notation, the infimum expression " $\left(A \wedge M_{\mathcal{L}}(t)\right)(x)$ " will be denoted by $M_{x}^{t}$.

Since every fuzzy set can be expressed as type-1 fuzzy set, see proposition 4.1, it is enough to state the following results in terms of type- 1 fuzzy sets. We assume that every type- 0 fuzzy set $r \in[0,1]$ is transformed into a type- 1 fuzzy set $C_{r}$ : $\{*\} \rightarrow[0,1]-$ where $\{*\}$ is a singleton and a special case of proposition $3.1-$ and every type-n fuzzy set, where $n>1$, is in its uncurried form.

It is not difficult to note that every function $M_{\mathcal{L}}(t)(u)$ has a connection with its domain. But, if you look closely to the fuzzification process, the infimum expression states that the same function $A(u)$ is used as operand together with every function $M_{\mathcal{L}}(t)(u)$. It means that the referred connection depends just on $U_{\mathcal{L}}$. So, each function $M_{\mathcal{L}}(t)(u)$ is associated with a type (its domain) $P_{u}$, no matter what $t \in$ $L\left(G_{\mathcal{L}}\right)$ is. Therefore, for each $u \in U_{\mathcal{L}}$, there is a family of functions

$$
\gamma(u)=\left\{M_{\mathcal{L}}(t)(u), A(u), M_{u}^{t}: P_{u} \rightarrow[0,1]\right\} .
$$

This fact enable us to "embed"each domain $P_{u}$ in a coerced domain $P$ which allow us to homogeneously apply supremum operation. This fact is revealed by the following proposition. 
Proposition 5.1. Given a linguistic variable $\mathcal{L}$, there is a family of functions

$$
\bar{\gamma}(u)=\left\{\overline{M_{\mathcal{L}}}(t)(u), \bar{A}(u), \bar{M}_{u}^{t}: P \rightarrow[0,1]\right\} .
$$

such that $\overline{M_{\mathcal{L}}}(t)(u), \bar{A}(u)$, and $\bar{M}_{u}^{t}$ are the respective coercions for $M_{\mathcal{L}}(t)(u), A(u)$, and $M_{u}^{t}$ of type $P_{u} \rightarrow[0,1]$ to the type $P \rightarrow[0,1]$; where $P$ is the direct product of every $P_{u}$.

Proof. Given $u \in U_{\mathcal{L}}$ and the associated family of type-1 fuzzy sets

$$
\gamma(u)=\left\{M_{\mathcal{L}}(t)(u), A(u), M_{u}^{t}: P_{u} \rightarrow[0,1]\right\} .
$$

Form the product

$$
P=\prod_{u \in U_{\mathcal{L}}} P_{u}
$$

For each $u \in U_{\mathcal{L}}$, define the following type-1 fuzzy sets: $\overline{M_{\mathcal{L}}}(t)(u), \bar{A}(u), \bar{M}_{u}^{t}$ : $P \rightarrow[0,1]$, such that

$$
\begin{aligned}
& \overline{M_{\mathcal{L}}}(t)(u)=M_{G}(t)(u) \circ \pi_{u} \\
& \bar{A}(u)=A(u) \circ \pi_{u} \\
& \bar{M}_{u}^{t}=M_{u}^{t} \circ \pi_{u} .
\end{aligned}
$$

By construction given $x \in P_{u}$, there is $\vec{z}$, such that

- $M_{\mathcal{L}}(t)(u)(x)=\overline{M_{\mathcal{L}}}(t)(u)(\vec{z})$,

- $A(u)(x)=\bar{A}(u)(\vec{z})$, and

- $M_{u}^{t}(x)=\overline{M_{u}^{t}}(\vec{z})$.

Moreover,

$$
\begin{aligned}
\overline{M_{u}^{t}}(\vec{z}) & =M_{u}^{t}\left(\pi_{u}(\vec{z})\right) \\
& =\left[M_{\mathcal{L}}(t)(u) \wedge A(u)\right]\left(\pi_{u}(\vec{z})\right) \\
& =\left[M_{\mathcal{L}}(t)(u)\left(\pi_{u}(\vec{z})\right) \wedge A(u)\left(\pi_{u}(\vec{z})\right)\right] \\
& =\left[\overline{M_{\mathcal{L}}}(t)(u)(\vec{z}) \wedge \vec{A}(u)(\vec{z})\right] \\
& =\left[\overline{M_{\mathcal{L}}}(t)(u) \wedge \bar{A}(u)\right](\vec{z}) .
\end{aligned}
$$

The equation (5.7) states the required coerced version of operands for the supremum expression at equation (5.1). This leads to the following theorem.

Theorem 5.1 (General Fuzzification Expression (GFE)). For every linguistic variable $\mathcal{L}$, there is a family of coersed functions $\bar{\gamma}(u)=\left\{\overline{M_{\mathcal{L}}}(t)(u), \bar{A}(u), \bar{M}_{u}^{t}: P \rightarrow\right.$ $[0,1]\}$ and the following coerced version for equation 5.1

$$
T_{\mathcal{L}^{A}}(t)=\bigvee_{u \in U_{\mathcal{L}}}\left(\bar{M}_{u}^{t}: P \rightarrow[0,1]\right)
$$

called here General Fuzzification Expression (GFE). 
Corollary 5.1.1. For every system of type-n and for every linguistic variable $\mathcal{L}$, the general fuzzification expression gives the following fuzzy set of type-2.

$$
T_{\mathcal{L}^{A}}: L(G) \rightarrow(P \rightarrow[0,1]) .
$$

Proof. Straightforward from the previous results, specially equation 5.8.

Observe that equation (5.9) gives a special case of the $T_{\mathcal{L}^{A}}$ in the definition 2.2 where all fuzzy sets denoted by $\mathbb{F} \mathbb{Z S E T}$ are just of the type $P \rightarrow[0,1]$ and all operations over fuzzy sets like infimum and supremum operations become homogeneous over that type. Observe also that $\mathbb{F} \mathbb{Z S E T}$ is a supertype which contains all possible types of the form $P \rightarrow[0,1]$.

\section{Final Remarks}

In this work we developed a mathematical investigation on linguistic variables and the process of fuzzification when it is taken into account heterogeneous linguistic variables. The most important result is equation 5.8 which specifies the fuzzification process in systems with different levels of uncertainty in the same linguistic variable.

The next step of this work is to investigate how other parts of an hetogeneous type-n system (like rules, defuzzification) looks like.

Resumo. Este trabalho propõe a generalização das noções de Variáveis Lingüísticas e do Processo de Fuzzificação. A idéia é fornecer um conceito de sistemas fuzzy no qual os termos lingüísticos de uma variável lingüística estejam, não necessariamente, ligados a conjuntos fuzzy de mesmo tipo. Tais sistemas serão chamados Sistema de Tipo-n. Nós introduzimos conceitos como Variáveis Lingüisticas $n$-Homogêneas que enfatizam a ocorrência de diferentes níveis de incerteza em tais Sistemas Fuzzy. O resultado mais importante é a proposição da Expressão de Fuzzificação Geral (GFE) que estabelece como o processo de fuzzificação deve ser calculado em qualquer sistema de tipo-n.

\section{References}

[1] G. Bojadziev, M. Bojadziev, "Fuzzy Sets, Fuzzy Logic, Applications", vol. 5, World Scientific, 1995.

[2] D.J. Dubois, H. Prade, "Fuzzy Sets and Systems: Theory and Applications", Academic Press, Inc., 1980.

[3] S. Fortune, J. Wyllie, Parallelism in random access machines. in "Proceedings of the tenth annual ACM symposium on Theory of Computing, STOC '78', pp. 114-118, ACM, New York, NY, USA, 1978.

[4] J. Hopcroft, R. Motwani, Jeffrey Ullman, "Introduction to Automata Theory, Languages, and Computation", Pearson/Addison Wesley, 2007. 
[5] G.J. Klir, B. Yuan, "Fuzzy Sets and Fuzzy Logics: Theory and Applications", Prentice Halls PTR, Upper Saddle River, NJ, 1995.

[6] K.H. Lee, "First Course On Fuzzy Theory And Applications", Springer-Verlag, 2004.

[7] W. Siler, J.J. Buckley, "Fuzzy Expert Systems and Fuzzy Reasoning", John Wiley \& Sons, 2005.

[8] A.M. Turing, On computable numbers, with an application to the entscheidungsproblem. Proceedings of the London Mathematical Society, s2-42, No. 1 (1937), 230-265.

[9] L.A. Zadeh, The concept of a linguistic variable and its application to approximate reasoning-i. Information Sciences, 8, No. 3 (1975), 199-249.

[10] L.A. Zadeh, The concept of a linguistic variable and its application to approximate reasoning-ii. Information Sciences, 8, No. 4 (1975), 301-357.

[11] L.A. Zadeh, The concept of a linguistic variable and its application to approximate reasoning-iii. Information Sciences, 9, No. 1 (1975), 43-80. 